\title{
Familial pellagra-like skin rash with neurological manifestations
}

\author{
E FREUNDLICH, M STATTER, AND S YATZIV
}

\author{
Department of Paediatrics, Government Hospital, Nahariya, Israel, and \\ Paediatric Research Unit and Department of Paediatrics, Hadassah University Hospital, Jerusalem, Israel
}

\begin{abstract}
SUMMARY A 14-year-old boy of Arabic origin presented with a pellagra-like rash and neurological manifestations including ataxia, dysarthria, nystagmus, and coma. There was a striking response to oral nicotinamide. The laboratory findings were not typical of Hartnup disease: aminoaciduria and indicanuria were absent and there was no evidence of tryptophan malabsorption. Tryptophan loading did not induce tryptophanuria nor did it increase excretion of xanthurenic or kynurenic acids. These findings support the possibility of a block in tryptophan degradation. The family history suggests a genetically-determined disorder.
\end{abstract}

We report a patient with familial pellagra-like skin rash, with neurological manifestations and laboratory findings that suggested a defect in the degradation of tryptophan.

\section{Case report}

A 14-year-old boy was the second child of healthy parents of Israeli-Arab origin who were 1st cousins. The mother had had 11 pregnancies. Two children had died, one at age 4 months after an attack of acute diarrhoea, the other at home at age 15 months, after having been admitted to hospital on several occasions with an undiagnosed skin condition, repeated and prolonged diarrhoea, and failure to thrive. An additional sibling had had a skin rash resembling pellagra at age 4 years; on another occasion he had had signs of ataxia lasting about one week.

The patient was admitted for the first time at age 13 months. A red, scaly rash resembling pellagra was distributed over the face, the upper portion of the thorax, and on the hands and legs. Routine laboratory examinations were normal as was the urinary amino-acid excretion. A diagnosis of pellagra was made and treatment with oral nicotinamide, $100 \mathrm{mg}$ daily, was initiated. The skin rash disappeared after several days.

The patient was readmitted to the hospital one year later with a similar skin condition and was again successfully treated with nicotinamide. During the following years the child's physical and mental development was normal, although the skin rash was noted several times. On one occasion a skin biopsy was performed and reported to be compatible with pellagra. A striking improvement was again noticed after nicotinamide administration.

The last admission was at age 14 years in late November (as were all previous admissions). At this time, he presented with mild confusion, diplopia, dysarthria, ataxia, and a pellagroid skin rash. His general condition deteriorated during the next few days; he became unable to walk or stand, and both horizontal and vertical nystagmus were observed. He became apathetic and entered into deep coma 4 days after admission. The electroencephalogram showed a markedly abnormal tracing with short bursts of high voltage 2.5 per second activity with superimposed sharp waves, mainly over the posterior region.

He was immediately treated with nicotinamide (100 mg daily) in view of the excellent results obtained on earlier occasions. On the third day of treatment his condition greatly improved. He regained consciousness and was able to walk by the fifth day of treatment. All neurological findings had returned to normal several days later. The electroencephalographic tracing, performed 6 days later, showed a marked improvement with runs of 3 per second activity over the posterior areas. Subsequent tracings confirmed a steady improvement and $\mathbf{3}$ months later the tracing was normal.

A presumptive diagnosis of Hartnup disease was made at this time because of the pellagra-like skin rash associated with severe neurological signs, as well as the abnormal electroencephalographic tracing and improvement after the treatment with nicotinamide. The normal urinary amino-acid excretion however, was not typical of this condition. It was therefore important to study the basic metabolic defect in this child and members of his family.

\section{Laboratory methods and results}

Urinary amino-acids. Screening of urine for free amino-acids was performed using unidimensional paper chromatography. Samples of urine containing $10 \mu \mathrm{l}$ creatinine were spotted on Whatman No 1 paper and chromatographed in butanol:acetic acid:water 
(60:15:20 by vol) and developed for 24 hours. $^{1}$ Urine was collected with a preservative (toluene) for 6-hour periods. Urine samples were analysed quantitatively for acidic and neutral amino-acid content on a $69 \times 0.9 \mathrm{~cm}$ column of PA-28 spherical cation exchange resin with Beckman model Unichrom amino-acid analyser. ${ }^{2}$ The paper chromatography showed a normal pattern which was also confirmed by quantitation of the amino-acid urinary content by amino-acid analyser (Table 1).

Tryptophan loading test. L-Tryptophan was given orally $(100 / \mathrm{mg}$ per $\mathrm{kg})$ and blood taken at 1 - or 2 hourly intervals for $\mathbf{8}$ hours. Plasma was prepared for quantitative tryptophan determination by the method of Mondino et al. ${ }^{3}$ Urinary kynurenic and xanthurenic acid levels were determined spectrofluorometrically. ${ }^{4}$ Intestinal absorption of tryptophan was normal or slightly increased in the patient and in one family member compared with a normal control of the same age.

Kynurenic and xanthurenic acids were examined in the urine before and after the tryptophan loading in the patient and in the normal control. They were found to be reduced in the patient by factors of 10 and 4 respectively (Table 2 ). These findings clearly suggest a disturbance in the normal metabolic degradation of tryptophan (Figure).

Table 1 Urinary amino-acid excretion ( $\mu \mathrm{mol} / \mathrm{min}$ per $1 \cdot 73 m^{2}$ )

\begin{tabular}{lll}
\hline Amino-acid & Patient & Normal range \\
\hline Threonine & 0.129 & $0.04-0.17$ \\
Serine & 0.423 & $0.09-0.34$ \\
Glutamine & 0.80 & $0.04-0.75$ \\
Glycine & 0.86 & $0.33-1 \cdot 5$ \\
Alanine & 0.54 & $0.04-0.35$ \\
Valine & 0.039 & trace-0.08 \\
Methionine & 0.04 & $0.01-0.04$ \\
Isoleucine & 0.013 & $0.01-0.07$ \\
Leucine & 0.113 & $0.02-0.11$ \\
Tyrosine & 0.13 & $0.03-0.12$ \\
Phenylalanine & 0.055 & $0.01-0.11$ \\
Ornithine & 0.019 & $0.01-0.03$ \\
Histidine & 0.58 & $0.11-1.0$ \\
\hline
\end{tabular}

Table 2 Urinary tryptophan metabolites (after loading)

\begin{tabular}{|c|c|c|c|c|c|}
\hline & \multicolumn{4}{|l|}{ Hours } & \multirow{2}{*}{$\begin{array}{c}\text { Total } \\
>24\end{array}$} \\
\hline & $-6-0$ & $0-6$ & $7-12$ & $13-24$ & \\
\hline \multicolumn{6}{|c|}{ Kynurenic acid } \\
\hline Patient & 0.005 & $1 \cdot 57$ & 0.88 & 0.040 & 2.49 \\
\hline Control & 0.009 & 19.0 & $1 \cdot 85$ & 0.028 & $20 \cdot 87$ \\
\hline \multicolumn{6}{|c|}{ Xanthurenic acid } \\
\hline Patient & 0.064 & 0.61 & 0.44 & $0 \cdot 134$ & $1 \cdot 18$ \\
\hline Control & 0.081 & $2 \cdot 51$ & 1.08 & $0 \cdot 140$ & $2 \cdot 72$ \\
\hline
\end{tabular}

All values are expressed as $\mu \mathrm{mol} / \mathrm{kg}$ per 6 hours.

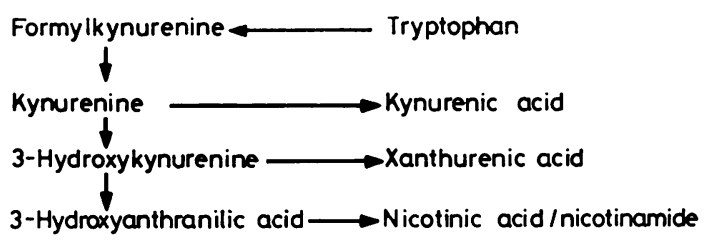

Figure Tryptophan degradation pathway.

Urinary indican. Urinary indican was determined by the method of Curzon and Walsh. ${ }^{5}$ Before and after tryptophan loading both in the patient and in other members of the family there were great variations, but no significant increase when compared with controls, especially if the results are expressed in $\mu \mathrm{mol} / \mathrm{kg}$ per $24 \mathrm{~h}$.

\section{Discussion}

Hartnup disease is characterised biochemically by aminoaciduria as well as by an intestinal defect in absorption of tryptophan, resulting in excessive indicanuria. Our patient presented several biochemical findings which differed from those described in Hartnup disease: (1) Hyperaminoaciduria (including tryptophanuria) was not found. (2) There was no evidence of tryptophan malabsorption, since oral tryptophan load showed a normal serum pattern of tryptophan and did not induce indicanuria. (3) After tryptophan loading there was neither tryptophanuria nor increased excretion of tryptophan metabolites in the form of xanthurenic and kynurenic acids.

Tada et al. ${ }^{6}$ reported a patient with a pellagroid rash and cerebellar ataxia, who also had congenital tryptophanuria, dwarfism, and mental retardation. A defect in conversion of tryptophan to kynurenine was suggested (Figure). Wong et al. ${ }^{7}$ described a patient with clinical features of Hartnup disease but without aminoaciduria and indicanuria, and without evidence of tryptophan malabsorption. An unusual sensitivity to nicotinic acid, absent in our patient, was present. A metabolic block in tryptophan oxidation was again suggested. Our case differs from that of Wong in that he did not show signs of persistent upper motor neuron abnormalities, and the cerebellar ataxia and other neurological signs soon responded to nicotinamide administration.

An inherited disease is suggested in our patient by the presence of similar symptoms in 2 siblings: one who died in infancy with an unusual skin rash and another who presented with a temporary ataxia and a pellagra-like rash. The parents were 1st cousins, so that an autosomal recessive inheritance seems probable. However, as all affected individuals were males sex-linked transmission cannot be excluded. 
The seasonal occurrence of clinical symptoms is well known in Hartnup disease. The skin rash is photosensitive and generally appears in the late summer. Our patient showed the same features; all the admissions to hospital occurred in November and neither a skin rash nor any other symptom was noted during other times of the year. Dietary tryptophan deficiency with decreased nicotinic acid production was not responsible for the clinical manifestations as the child regularly received a diet containing sufficient tryptophan.

The cause of the neurological disturbances in patients with the classical form of Hartnup disease has not been clearly established. Most doctors believe that the high content of tryptophan in the intestine gives rise to an increased quantity of degradation products by the intestinal flora which are absorbed into the blood stream and are responsible for the neurological symptoms. In our patient the mechanism seems to be different, since the absorption of the tryptophan from the jejunum was normal. It may therefore be assumed that either a production of an abnormal by-product or deficiency of a metabolic end-product in tryptophan degradation pathway (Figure) is the reason for the neurological and other clinical signs in our case. The striking response of these patients to administration of nicotinamide favours the possibility of a metabolic block in tryptophan degradation.
We thank Mrs A Shina and Miss G Barfi for technical assistance, and $\operatorname{Dr} \mathrm{Y}$ Manelis for the neurological evaluation.

\section{References}

1 Smith J. Amino acids, amines, and related compounds. In: Smith J, ed. Chromatographic techniques. London: Heinemann, 1963: 82-142.

2 Spackman D H, Stein W H, Moore S. Automatic recording apparatus for use in the chromatography of amino acids. Anal Chem 1958; 30: 1190-206.

8 Mondino A, Bongiovanni G, Fumero S. A new approach for obtaining total tryptophan recovery in plasma samples deproteinised with sulfosalicilic acid. J Chromatogr 1975; 104: 297-302.

4 Price J M, Brown R R, Yess N. Testing the functional capacity of the tryptophan-niacin pathway in man by analysis of the urinary metabolites. Adv Metab Disord $1965 ; 2$ : 159-225.

5 Curzon G, Walsh J. A method for the determination of the urinary indoxyl sulphate (indican). Clin Chim Acta 1962; 7: 657-63.

- Tada K, Ito H, Arakawa T. Congenital tryptophanuria with dwarfism (' $H$ ' disease-like clinical features without indicanuria and general amino-aciduria): a probably new inborn error of tryptophan metabolism. Tohoku J Exp Med 1963; 80: 118-34.

7 Wong P W, Forman P, Tabahoff B, Justice P. A defect in tryptophan metabolism. Pediatr Res 1976; 10: 725-30.

Correspondence to Dr S Yatziv, Department of Paediatrics, Hadassah University Hospital, PO Box 12000, il-91 120, Jerusalem, Israel.

Received 11 March 1980

\title{
Rigid spine syndrome and fatal cardiomyopathy
}

\author{
A F COLVER, C R STEER, M J GODMAN, AND W S UTTLEY \\ Royal Hospital for Sick Children and Department of Child Life and Health, Edinburgh
}

\begin{abstract}
SUMMARY A $7 \frac{1}{2}$-year-old girl had the clinical features of the rigid spine syndrome of Dubowitz. Muscle biopsy showed a predominance of type 2 fibres with neither myopathic features nor an increase in connective tissue. In addition, she had a hypertrophic cardiomyopathy with which she presented in heart failure and from which she died suddenly one month later. The association of rigid spine syndrome with cardiomyopathy has not been reported previously.
\end{abstract}

We record the unusual combination of the rigid spine syndrome of Dubowitz and fatal cardiomyopathy.

\section{Case report}

A girl, aged $7 \frac{1}{2}$ years, presented with breathlessness on exercise and increasing tiredness over 2 months. She had had no blue attacks, chest pain, or fainting, nor had she any other complaint; she had attended school normally. She was the first child of unrelated parents. Pregnancy and birth had been normal. Her two siblings were normal. There was no family history of neuromuscular or cardiac disease. At 5 years, she had been noted to have an extended neck, restricted spinal movement, and to be thin.

She was very thin (Fig. 1). There was loss of subcutaneous fat with relative sparing of the face. Her height was average for her age and greater than her 